OPEN ACCESS

Edited by:

Michael Kaess,

University of Bern, Switzerland

Reviewed by:

Amy Marie Brausch,

Western Kentucky University,

United States

Glenn Kiekens,

KU Leuven, Belgium

Marc Schmid,

Universitäre Psychiatrische Kliniken,

Switzerland

*Correspondence:

Maria Zetterqvist

maria.zetterqvist@liu.se

Specialty section:

This article was submitted to Child and Adolescent Psychiatry,

a section of the journal

Frontiers in Psychiatry

Received: 25 July 2019

Accepted: 06 January 2020

Published: 14 February 2020

Citation:

Zetterqvist M, Perini I, Mayo LM and Gustafsson PA (2020) Nonsuicidal Self-Injury Disorder in Adolescents:

Clinical Utility of the Diagnosis Using the Clinical Assessment of Nonsuicidal

Self-Injury Disorder Index.

Front. Psychiatry 11:8

doi: 10.3389/fpsyt.2020.00008

\section{Nonsuicidal Self-Injury Disorder in Adolescents: Clinical Utility of the Diagnosis Using the Clinical Assessment of Nonsuicidal Self- Injury Disorder Index}

\author{
Maria Zetterqvist ${ }^{1,2 *}$, Irene Perini ${ }^{1}$, Leah M. Mayo ${ }^{1}$ and Per A. Gustafsson ${ }^{1,2}$ \\ ${ }^{1}$ Center for Social and Affective Neuroscience, Department of Clinical and Experimental Medicine, Linköping University, \\ Linköping, Sweden, ${ }^{2}$ Department of Child and Adolescent Psychiatry, Region Östergötland, Linköping, Sweden
}

Nonsuicidal self-injury disorder (NSSID) is a condition in need of further study, especially in adolescent and clinical populations where it is particularly prevalent and studies are limited. Twenty-nine clinical self-injuring adolescents were included in the study. The Clinical Assessment of Nonsuicidal Self-Injury Disorder Index (CANDI) was used to assess prevalence of NSSID. The NSSID diagnosis criteria were met by $62.1 \%$ of adolescents. The impairment or distress criterion was least often met. Criteria B and C (assessing reasons for NSSI and cognitions/emotions prior to NSSI) were confirmed by $96-100 \%$ of all participants. Adolescents with NSSI in this clinical sample had several comorbidities and high levels of psychopathology. NSSID occurred both in combination with and independently of borderline personality disorder traits as well as suicide plans and attempts. Those with NSSID had a significantly higher cutting frequency than those not meeting full NSSID criteria. Other NSSI characteristics, comorbidity, psychopathology, and trauma experiences did not differ between groups. CANDI was a feasible tool to assess NSSID in adolescents. It is important to use structured measures to assess the validity of the NSSID diagnosis across development in both community and clinical samples. The clinical utility of the NSSID diagnosis is discussed.

Keywords: nonsuicidal self-injury, disorder, adolescents, Diagnostic and Statistical Manual of Mental Disorders, diagnosis

\section{INTRODUCTION}

Nonsuicidal self-injury (NSSI), i.e., deliberately injuring one's own body tissue without suicidal intent (1), is a significant mental health problem among adolescents. This is especially true in clinical populations, in which $40 \%$ or more report NSSI (2). NSSI is also common in non-clinical samples, with prevalence rates estimated to be around $18 \%$ (3). There is an ongoing discussion whether NSSI should be considered a separate diagnostic entity (4). Those in favor of a nonsuicidal self-injury disorder (NSSID) diagnosis emphasize the pros of having specified criteria cut-offs. This would enable identification of individuals with more severe NSSI who potentially are at high risk 
and in need of treatment. Furthermore, a diagnosis would stimulate treatment research and distinguish the condition from borderline personality disorder (BPD) and suicidality. It would also lead to improved communication and conceptual clarity in clinical practice $(5,6)$.

In 2013, NSSI was included in section III of the fifth version of the Diagnostic and Statistical Manual of Mental Disorders [DSM-5; (7)], as a condition in need of further study, making it a highly relevant research area. As currently proposed, NSSID is a dichotomous diagnosis (8), consisting of six criteria that all have to be met in order for a diagnosis of NSSID to be applicable. The potential diagnosis of NSSID is conceptualized as a condition that can occur with or without other comorbidities, such as BPD, as well as suicidality (9). Preliminary data have shown that NSSID prevalence rates range between $5.6 \%$ and $7.6 \%$ among non-clinical samples of adolescents (10-12), and $0.2-0.8 \%$ in young adults $(13,14)$. In clinical adolescent selfinjuring samples, between $74 \%$ and $78 \%$ meet full criteria $(8,15)$.

Since the criteria were published, they have been subject to discussion (e.g., 6, 8, 16-19). Based on recent empirical data, revisions of the criteria have been suggested, including proposals that a dimensional approach to the disorder would be more beneficial (8). Criterion A is a frequency criterion, with NSSI occurring on at least 5 days during the past year. See Table 1 for full NSSID diagnostic criteria (7). The validity of criterion A has been examined and debated. Recent studies suggest that the cutoff level needs to be increased and/or the time period needs to be decreased to better delimit individuals with more severe NSSI (16-18). Furthermore, severity needs to be taken into account (16-18). Currently, no distinction is made based on severity between five incidents of less serious damage to body tissue by scratching, for instance, and five episodes of severe cutting that need to be stitched. Criteria B and C measure reasons for engaging in NSSI and emotions/cognitions experienced prior to NSSI, respectively. Criterion C further measures preoccupation with and thoughts about NSSI that are difficult to resist that occur before NSSI. Criterion B has shown to be very highly endorsed and does not discriminate sufficiently since it is overinclusive $(8,20)$. Similarly, criterion C, specifically C1, has shown to be very commonly endorsed, with interpersonal difficulties or negative states preceding the NSSI incident (8). In a study by Washburn and colleagues (8), criterion C1 was significantly associated with several measures of psychopathology and impairment.

Criterion D ensures that behaviors that are considered socially sanctioned or minor are not included. The diagnostic prerequisite of functional impairment and/or distress caused by the disorder is met by criterion E. The issue of impairment or distress has also been empirically examined (21). Although criterion $\mathrm{E}$ is often the least commonly met in several studies $(11,14,21,22)$, it best discriminates those with an NSSID diagnosis from those not meeting full criteria (23). Finally, criterion $\mathrm{F}$ describes possible exclusion and differential diagnoses that potentially better explain the self-injurious behavior (7).
TABLE 1 | Proposed Nonsuicidal Self-Injury Criteria in DSM-5.

A. In the last year, the individual has, on 5 or more days, engaged in intentional self-inflicted damage to the surface of his or her body of a sort likely to induce bleeding, bruising, or pain (e.g., cutting, burning, stabbing, hitting, excessive rubbing), with the expectation that the injury will lead to only minor or moderate physical harm (i.e., there is no suicidal intent).

Note: The absence of suicidal intent has either been stated by the individual or can be inferred by the individual's repeated engagement in a behavior that the individual knows, or has learned, is not likely to result in death.

B. The individual engages in the self-injurious behavior with one or more of the following expectations:

1. To obtain relief from a negative feeling or cognitive state.

2. To resolve an interpersonal difficulty.

3. To induce a positive feeling state.

Note: The desired relief or response is experienced during or shortly after the self-injury, and the individual may display patterns of behavior suggesting a dependence on repeatedly engaging in it.

C. The intentional self-injury is associated with at least one of the following:

1. Interpersonal difficulties or negative feelings or thoughts, such as depression, anxiety, tension, anger, generalized distress, or self-criticism, occurring in the period immediately prior to the self-injurious act.

2. Prior to engaging in the act, a period of preoccupation with the intended behavior that is difficult to control.

3. Thinking about self-injury that occurs frequently, even when it is not acted upon.

D. The behavior is not socially sanctioned (e.g., body piercing, tattooing, part of a religious or cultural ritual) and is not restricted to picking a scab or nail biting.

E. The behavior or its consequences cause clinically significant distress or interference in interpersonal, academic, or other important areas of functioning.

F. The behavior does not occur exclusively during psychotic episodes, delirium, substance intoxication, or substance withdrawal. In individuals with a neurodevelopmental disorder, the behavior is not part of a pattern of repetitive stereotypies. The behavior is not better explained by another mental disorder or medical condition (e.g., psychotic disorder, autism spectrum disorder, intellectual disability, Lesch-Nyhan syndrome, stereotypic movement disorder with self-injury, trichotillomania [hair-pulling disorder], excoriation [skin-picking] disorder)

Reprinted with permission from the Diagnostic and Statistical Manual of Mental Disorders Fifth Edition, (Copyright @2013). American Psychiatric Association.

The main objective of the diagnosis, i.e., to identify individuals with more severe NSSI, has been examined. Studies that have tried to validate the NSSID diagnosis have shown that it exists both independently of BPD and other disorders (24), as well as comorbidly $(8,15)$. The criteria can also detect a more severe group compared to individuals with NSSI who do not meet diagnostic criteria. Those with NSSID show greater NSSI versatility, as well as more psychopathology and functional impairment $(8,14,15,22,23,25)$. Studies that have examined whether the NSSID criteria could demarcate individuals from those with NSSI in clinical adolescent samples $(8,25)$ have shown preliminary support for more suicidality in the NSSID group, but also some inconsistencies. The clinical utility of the current diagnostic criteria in clinical samples has also been questioned (8).

Earlier empirical research on NSSID has predominately been done on (young) adults and/or in community/college samples. Only very few studies have focused on clinical and adolescent populations $(8,15,18,25)$. Thus, further research is sorely needed to test the clinical utility of the NSSID diagnosis, as 
clinical adolescents are those most likely to receive a diagnosis and need treatment planning in clinical practice. The clinical utility of the proposed NSSID criteria is its ability to reliably identify and delimit a group of individuals who are suffering and are impaired by their NSSI. Individuals that have more severe symptoms and risk a negative developmental trajectory are in need of specific treatment.

NSSI characteristics such as methods, frequency and age of onset, clinical psychopathology including comorbid psychiatric diagnoses, BPD, suicidality, emotional awareness and difficulties with emotion regulation, negative life events, and trauma experience are possible variables that are relevant in discriminating between NSSID and NSSI and in examining the clinical utility of the NSSID criteria $(26,27)$.

Several earlier studies on NSSID have used self-report or other different measures to operationalize the criteria, making comparisons difficult. Clinical interviews developed to assess the NSSID diagnosis have been lacking. The clinical trials preceding DSM-5 resulted in lack of reliability for the NSSID diagnosis (28), which indicates that reliable and valid measures to assess NSSID are crucial to advance the research instead of relying solely on self-report. One such addition is the Clinical Assessment of Nonsuicidal Self-injury Disorder Index (CANDI; 23), which is a semi-structured clinical interview that assesses the full NSSID criteria. CANDI was originally developed for adults and has been psychometrically validated on an adult population (23). The Swedish version has also been used to assess adolescents that participated in an emotion regulation treatment study $(29,30)$, suggesting that it has potential for this population. There is a need for studies that examine the feasibility of structured measures to assess NSSID, especially in psychiatric adolescent populations, in order to validate and document NSSID prevalence across development, to identify risk and facilitate treatment planning.

To conclude, previous research on the NSSID diagnosis has focused on adult samples, and there are few studies on NSSID in clinical adolescent samples. Structured diagnostic interviews are important for assessment, but these are lacking and little research has been done, especially on psychiatric samples. Advances within this field will help clinicians adequately assess NSSID and avoid over-pathologizing the behavior. Early identification of NSSID may support intervention strategies for those in need and prevent a further negative development of NSSI.

The aim of this explorative pilot study was to investigate the feasibility of assessing DSM-5 NSSID criteria using the structured interview CANDI in a clinical sample of adolescents by examining whether a more severe group could be identified by comparing demographics, NSSI characteristics, psychopathology, and trauma experience of those with an NSSID diagnosis to those with NSSI not meeting full NSSID criteria.

\section{METHOD}

\section{Participants and Procedure}

Participants with NSSI were recruited from the child and adolescent psychiatric (CAP) clinic in Linköping, Sweden, from June 2016 to March 2018, as part of a study examining neurobiological markers of NSSI in 30 clinical and 30 healthy adolescents which was carried out at the Center for Social and Affective Neuroscience, Linköping University (31). Inclusion criteria for participants were: having engaged in five or more instances of NSSI during the past 6 months, independent of psychiatric diagnosis, and being a female between 15 and 18 years of age. The time period was limited to 6 months to ensure that participants had relatively recent NSSI, since temporal proximity is important when answering questions referring to NSSI characteristics (such as frequency, methods, and functions), in order avoid questionable retrospective recall (32). The lower age limit was set to 15 years to increase chances of obtaining valid responses from measures, some of which were originally developed for adults. We also limited the sample to females since NSSI is more prevalent in females in clinical samples (33). Based on clinical experience we predicted that the sample would include only one to two male participants if males were included. Exclusion criteria were: current or lifetime diagnosis of schizophrenia, bipolar or psychotic disorder and/or alcohol/drug dependence, and IQ below 80. Adolescents meeting inclusion criteria were approached with oral and written information about the study. Participants (and parents, if the participant was $<18$ years) gave written informed consent. The study was approved by the Regional Ethical Board of Linköping (Dnr 2015/273-31; 2016/224-32).

Participants were assessed at the CAP clinic during one or two sessions by the first author, a clinical child psychologist with extensive experience in psychiatric assessment, who evaluated the NSSID diagnosis. Final comorbid psychiatric diagnoses for the clinical sample were made together with the last author, a child psychiatrist, and were based on all available information from diagnostic interviews and medical records, using DSM-5 (7).

Of the 30 adolescents with NSSI that were recruited to the study, one was not assessed with CANDI. Thus, 29 female participants $(M=15.9$ years, $S D=0.77)$ were included in the present study.

\section{Psychometric Measures Demographics}

Demographic information concerning family status and living conditions, ethnicity and parental education was assessed with questions developed for this study, and from clinical records

\section{Nonsuicidal and Suicidal Self-Injury Thoughts and Behaviors}

NSSI characteristics (including frequency and means of selfinjury) and diagnosis were assessed using the Clinical Assessment of Nonsuicidal Self-injury Disorder Index (CANDI; 23), which is a semi-structured clinical interview that assesses the full NSSID criteria. CANDI includes an initial screen of past year NSSI, both frequency and days. Each diagnostic criterion is assessed using yes/no presence of the criterion and continuous follow-up data are obtained. CANDI was originally developed for adults, and has been psychometrically validated on 
an adult population demonstrating good interrater reliability and adequate internal consistency (23). The Swedish version has recently been used to assess adolescents who participated in an emotion regulation treatment study $(29,30)$.

Selected questions from the semi-structured Self-Injurious Thoughts and Behaviors Interview (SITBI; 34) were used to obtain detailed information about suicidal behavior. SITBI assesses the presence, frequency, and characteristics of selfinjurious thoughts and behaviors, and has psychometrically shown strong interrater reliability, test-retest reliability, and concurrent validity (34). SITBI has also been validated in Swedish with good psychometric properties (12).

\section{Psychopathology}

The clinical interview Schedule for Affective Disorders and Schizophrenia for School-Age Children-Present and Lifetime Version (K-SADS-PL; 35) from 2009 was used for DSM-IV diagnoses. K-SADS-PL has three sections: 1) introductory interview; 2) screen interview; 3) eight optional supplements. These refer to: 1) affective disorders; 2) psychotic disorders; 3 ) anxiety disorders; 4) behavioral disorders; 5) substance use disorders; 6) eating disorders; 7) tic disorders; 8) autism spectrum disorders.

Difficulties in Emotion Regulation Scale (DERS; 36) was used to measure difficulties in identifying and regulating negative emotions and difficulties in using goal-directed behavior while under the influence of negative emotions. The questionnaire consists of 36 items rated on a 5-point Likert-type scale from "almost never" to "almost always." It has six subscales: difficulties in emotional acceptance, difficulties in goal-directed behavior, difficulties in impulse control, lack of emotional awareness, lack of emotion regulating strategies, and lack of emotional clarity. Higher scores indicate more difficulties with emotion regulation. The questionnaire has been used both clinically and in normative populations for adults and adolescents, and has shown to have high internal consistency, good test-retest reliability, and adequate construct validity. Cronbach's alpha for the clinical adolescents' total score in the present sample was $\alpha=0.78$, indicating acceptable internal consistency.

Toronto Alexithymia Scale (TAS-20; 37) was used to assess alexithymia. TAS-20 has 20 items, ranging from totally right to totally wrong on a 5-grade Likert-type scale. The questionnaire comprises three subscales: difficulties identifying emotions, difficulties describing emotions, and difficulties externalizing emotions. Higher scores indicate higher levels of alexithymia. TAS-20 is one of the most commonly used self-report scales for alexithymia and has shown good reliability and validity. In the present clinical adolescent sample, internal consistency for the total TAS-20 was $\alpha=0.70$; which is acceptable.

\section{Borderline Personality Traits}

The Structural Clinical Interview for DSM-IV Personality Disorders (SCID-II; 38) was administered for symptoms of borderline personality disorder (BPD). Both the self-report and interview versions were used in the present study. Cronbach's alpha for the self-report version of BPD was $\alpha=0.85$ in the present sample.

\section{Traumatic Experiences}

The Trauma Symptom Checklist for Children (TSCC; 39) is a self-report questionnaire developed to identify symptoms of traumatic stress in children and adolescents. The questionnaire consists of 54 items and the respondents rate their answers on a 4 -point Likert scale from 0 (never) to 3 (almost always). The results are divided into six subscales (depression, anxiety, dissociation, post-traumatic stress, sexual concern, and anger). TSCC has been evaluated on Swedish children and adolescents with good reliability (internal consistency, test-retest) and satisfactory concurrent and criterion-related validity (40). TSCC showed excellent internal consistency for the total scale in the present sample, $\alpha=0.92$.

Linköping Youth Life Experience Scale (LYLES; 41) is an instrument for gauging potentially traumatic life events, including adverse childhood circumstances. LYLES contains 23 main questions and includes measures of interpersonal, noninterpersonal, and adverse childhood circumstances. LYLES has been evaluated on Swedish adolescents from the normative population. Its psychometric properties have been shown to be satisfactory (41).

\section{Intelligence}

An abbreviated version of Wechsler Intelligence Scales, fourth edition for children (42) or adults (43), was used to assess intelligence depending on participants' age.

\section{Data Analysis}

Continuous data were first tested for normality using ShapiroWilk. NSSI frequency and reported NSSI reasons and experiences prior to NSSI were not normally distributed. Data were analyzed with descriptive statistics using frequencies, mean values, cross-tabulation with chi-square $\left(\chi^{2}\right)$ and independent samples t-tests. All data were baseline data. For data not meeting requirements for normal distribution, between group comparisons were analyzed with nonparametric MannWhitney $U$ test and median was presented. For crosstabulation of categorical data, Fischer's exact test was used when cells had an expected count less than five. Internal consistency was assessed using Cronbach's alpha $(\alpha)$ for the self-report measures. All statistical analyses were performed using the SPSS 24.0 software package (SPSS Inc, Chicago, IL).

\section{RESULTS}

\section{Demographic Characteristics}

All participating adolescents were female $(n=29,100.0 \%)$. Mean age (SD) was 15.9 (0.77) years for the total NSSI group. Adolescents meeting full criteria for the NSSID diagnosis had a significantly higher IQ $(M=99.0, S D=8.25)$ compared to those not meeting full criteria $(M=91.0, S D=9.45)$. There were no significant differences between groups concerning age, family structure, parental education, or ethnicity. Sample characteristics are presented in Table 2 . 
TABLE 2 | Participant demographics.

\begin{tabular}{|c|c|c|c|c|}
\hline $\begin{array}{l}\text { Demographic } \\
\text { characteristics }\end{array}$ & $\begin{array}{c}\text { Total NSSI } \\
\text { sample } \\
\begin{array}{c}n=29 \\
n(\%)\end{array}\end{array}$ & $\begin{array}{c}\text { NSSID } \\
n=18 \\
n(\%)\end{array}$ & $\begin{array}{c}\text { NSSI } \\
n=11 \\
n(\%)\end{array}$ & $p$ \\
\hline \multicolumn{5}{|l|}{ Sex } \\
\hline Female & $29(100.0)$ & $18(100.0)$ & $11(100.0)$ & - \\
\hline \multicolumn{5}{|l|}{ Age } \\
\hline$m(S D)$ & $15.9(0.77)$ & $15.8(0.86)$ & $16.0(0.63)$ & $0.58 \neq$ \\
\hline \multicolumn{5}{|l|}{$\mathrm{IQ}$} \\
\hline$m(S D)$ & $96.0(9.42)$ & $99.0(8.25)$ & $91.0(9.45)$ & $0.02 \neq$ \\
\hline Family structure & & & & $1.0 \dagger$ \\
\hline Married/co-habitant & $12(41.4)$ & 7 (38.9) & $5(45.5)$ & \\
\hline Divorced & $17(58.6)$ & $11(61.1)$ & $6(54.5)$ & \\
\hline $\begin{array}{l}\text { Mother's highest level of } \\
\text { education }\end{array}$ & $n=28$ & $n=17$ & $n=11$ & $0.41 \leftarrow$ \\
\hline University/college & $13(46.4)$ & $8(47.1)$ & $5(45.4)$ & \\
\hline $\begin{array}{l}\text { Theoretical high school } \\
\text { program }\end{array}$ & $2(7.1)$ & $2(11.8)$ & $0(0.0)$ & \\
\hline $\begin{array}{l}\text { Vocational high school } \\
\text { program }\end{array}$ & $12(42.9)$ & $7(41.2)$ & $5(45.4)$ & \\
\hline Compulsory school & $1(3.6)$ & $0(0.0)$ & $1(9.1)$ & \\
\hline $\begin{array}{l}\text { Father's highest level of } \\
\text { education }\end{array}$ & $n=25$ & $n=16$ & $n=9$ & $0.62 \downarrow$ \\
\hline University/college & $9(36.0)$ & $6(37.5)$ & 3 (3.33) & \\
\hline $\begin{array}{l}\text { Theoretical high school } \\
\text { program }\end{array}$ & $2(8.0)$ & $1(6.3)$ & $1(11.1)$ & \\
\hline $\begin{array}{l}\text { Vocational high school } \\
\text { program }\end{array}$ & $11(44.0)$ & $8(50.0)$ & 3 (33.3) & \\
\hline Compulsary school & $3(12.0)$ & $1(6.3)$ & $2(22.2)$ & \\
\hline Parent born in other country & $\begin{array}{l}n=55 \\
4(7.3)\end{array}$ & $\begin{array}{l}n=35 \\
4(11.4)\end{array}$ & $\begin{array}{l}n=20 \\
0(0.0)\end{array}$ & $0.28+$ \\
\hline
\end{tabular}

NSSI, nonsuicidal self-injury; NSSID, nonsuicidal self-injury disorder.

\# Independent sample t-test. † Fischer's exact test. \& Pearson's chi-square.

TABLE 4 | Self-injury characteristics and comparisons between NSSI groups.

\begin{tabular}{lcccc}
\hline Measure & $\begin{array}{c}\text { Total NSSI } \\
\text { sample }\end{array}$ & NSSID & NSSI & $p$ \\
$(n=29)$ & & & \\
$(n=18)$ & & & \\
& & &
\end{tabular}

TABLE 3 | Participants meeting NSSID criteria, frequencies and percentages.

\begin{tabular}{lccc}
\hline Criteria & $\begin{array}{c}\text { Total NSSI sample } \\
(\boldsymbol{n}=\mathbf{2 9 )}\end{array}$ & $\begin{array}{c}\text { NSSID } \\
(\boldsymbol{n}=\mathbf{1 8})\end{array}$ & $\begin{array}{c}\text { NSSI } \\
(\boldsymbol{n}=\mathbf{1 1})\end{array}$ \\
\hline Criterion A & $25(86.2)$ & $18(100.0)$ & $7(63.6)$ \\
Criterion B & $28(96.6)$ & $18(100.0)$ & $10(90.9)$ \\
Criterion C & $29(100.0)$ & $18(100.0)$ & $11(100.0)$ \\
Criterion D & $29(100.0)$ & $18(100.0)$ & $11(100.0)$ \\
Criterion E & $21(72.4)$ & $18(100.0)$ & $3(27.3)$ \\
Criterion F & $29(100.0)$ & $18(100.0)$ & $11(100.0)$ \\
NSSID diagnosis & $18(62.1)$ & $18(100.0)$ & $0(0.0)$ \\
\hline
\end{tabular}

NSSID, nonsuicidal self-injury disorder.

\section{Self-Injury Characteristics and Comparisons Between NSSI Groups}

Of the total sample of adolescents with NSSI, 18 (62.1\%) met full criteria for NSSID according to CANDI, while 11 (37.9\%) did not. For the proportion of adolescents meeting NSSID criteria see Table 3. Criterion A was met by $86.2 \%$ of the total sample. Those not meeting criterion A (13.8\%), despite the presence of five or more NSSI episodes on different days during the past year, were assessed as having too minor a form of self-injury that did

\section{NSSI}

Age of onset $m(S D)$

Number of methods

$m$ (SD)

12 months cutting frequency

$m(S D)$, median

12 months severely scratched

frequency

$m(S D)$, median

12 months prevented wounds

from healing frequency

$m$ (SD), median

12 months punched oneself

frequency

$m(S D)$, median

Three most commonly

$100 \%$

$m(S D)$, median

(1)

Three most commonly endorsed emotion/cognition prior to NSSI 0-100\% $m(S D)$, median endorsed reasons for NSSI O-

\begin{tabular}{|c|c|c|c|}
\hline $13.2(1.25)$ & $12.9(1.34)$ & $13.6(1.03)$ & $0.16 \neq$ \\
\hline $3.76(2.13)$ & $4.17(2.26)$ & 3.09 (1.81) & $0.19 \neq$ \\
\hline 64.28 & 94.94 & 14.09 & $0.001^{*}$ \\
\hline (83.36) & (93.55) & (10.77) & \\
\hline 30.0 & 75.0 & 10.0 & \\
\hline 15.07 & 22.11 & 3.55 & $0.16^{*}$ \\
\hline (29.53) & (37.74) & (5.92) & \\
\hline 2.0 & 4.0 & 0.0 & \\
\hline 33.72 & 51.78 & 4.18 & $0.13^{*}$ \\
\hline (79.39) & $(97.20)$ & (6.19) & \\
\hline 1.0 & 12.5 & 0.0 & \\
\hline 15.55 & 21.94 & 5.09 & $0.98^{*}$ \\
\hline (56.18) & (70.87) & (10.20) & \\
\hline 0.0 & 0.0 & 0.0 & \\
\hline Decrease & Decrease & Decrease & $0.73^{*}$ \\
\hline negative & negative & negative & \\
\hline emotion & emotion & emotion & \\
\hline 80.69 & 87.78 & 69.09 & \\
\hline (25.45) & (9.27) & $(37.80)$ & \\
\hline 90.0 & 90.0 & 90.0 & \\
\hline Punish & Punish & Punish & $0.62^{*}$ \\
\hline 70.52 & 72.22 & 67.73 & \\
\hline (33.87) & (33.35) & (36.15) & \\
\hline 90.0 & 90.0 & 70.0 & \\
\hline Distract & Distract & Distract & $0.50^{*}$ \\
\hline 51.55 & 48.33 & 56.82 & \\
\hline (34.26) & (33.12) & (37.08) & \\
\hline 50.0 & 50.0 & 50.0 & \\
\hline $\mathrm{Sad} /$ & $\mathrm{Sad} /$ & Sad/ & $0.76^{\star}$ \\
\hline depressed & depressed & depressed & \\
\hline 79.93 & 80.72 & 78.64 & \\
\hline (23.68) & (24.21) & (23.88) & \\
\hline 90.0 & 91.50 & 90.0 & \\
\hline Worthless & Worthless & Worthless & $0.28^{*}$ \\
\hline 74.38 & 72.06 & 78.18 & \\
\hline (28.51) & (27.79) & $(30.60)$ & \\
\hline 90.0 & 80.0 & 100.0 & \\
\hline Failure & Failure & Failure & $0.55^{\star}$ \\
\hline 72.90 & 70.22 & 77.27 & \\
\hline (22.40) & (25.08) & (17.37) & \\
\hline 80.0 & 75.0 & 80.0 & \\
\hline 29 (100.0) & $18(100.0)$ & $11(100.0)$ & - \\
\hline $15(51.7)$ & $11(61.1)$ & $4(36.4)$ & $0.36+$ \\
\hline $10(34.5)$ & $7(38.9)$ & $3(27.3)$ & $0.69+$ \\
\hline
\end{tabular}

Suicidal behaviors

Suicide ideation

Frequency (\%)

Suicide plans

Frequency (\%)

Suicide attempt

Frequency (\%)

NSSI, nonsuicidal self-injury; NSSID, nonsuicidal self-injury disorder. \# Independent sample t-test (due to small samples analyses were also run with Mann-Whitney U-test with similar results). * Nonparametric Mann-Whitney U-test. † Fischer's exact test.

not result in bleeding or bruising. All participants (100.0\%) met criteria C, D and F. All but one participant (96.6\%) met criterion $\mathrm{B}$, as assessed by CANDI, since she did not confirm any of the suggested or other reasons for engaging in NSSI (Table 4). The criterion that was met least often was criterion $\mathrm{E}$, with self-injury being associated with suffering or impairment for $72.4 \%$ in this 
sample. The 21 adolescents (72.4\%) that endorsed criterion E mostly acknowledged impairment/distress due to having to cover up scars during summertime or experiencing shame and guilt over cutting, as well as worrying about family members' reactions to their NSSI. For adolescents that did not meet criterion E, distress or impairment was not attributed to NSSI per se, but rather to symptoms associated with other diagnoses or problems.

Mean age of onset for NSSI was $12.9(S D=1.34)$ years in the NSSID group $(n=18)$ compared to $13.64(S D=1.03)$ years in the NSSI group not meeting full criteria $(n=11)$. Mean number of different NSSI methods used in the total sample was 3.76 (2.13). There were no differences between groups regarding age of onset or number of methods. The only significant difference in selfinjury characteristics between groups was the 12-month cutting frequency $(p=0.001)$, where those with NSSID had a significantly higher frequency than those not meeting full NSSID criteria. Other methods of self-injury, such as severely scratching, punching oneself, or preventing wounds from healing, did not differ between groups. See Table 4. The three most commonly reported reasons for engaging in NSSI (criterion B) were the same in both groups: to decrease or relieve a negative emotion; to punish yourself; to distract yourself from negative thoughts or feelings. The three most commonly reported emotions and thoughts that were experienced before engaging in NSSI (criterion C) were also the same in both groups: feeling sad, depressed or down; feeling worthless, hopeless, or undeserving; and feeling like a failure or inferior (Table 4). There were no differences between groups regarding reported reasons or experiences prior to NSSI. There was considerable cooccurrence of NSSI and suicidality in the present sample. Every adolescent with NSSI $(n=29,100.0 \%)$ reported life-time prevalence of suicidal ideation. Suicidal plans were reported by $61.1 \%$ of those with NSSID and $36.4 \%$ in the NSSI group, but the difference was not statistically significant. Suicide attempts were reported by $34.5 \%$ in the NSSID group, and $27.3 \%$ of those with NSSI reported having made a suicide attempt. The difference was not significant. See Table 4.

The CANDI interview was feasible to administer to this clinical sample of adolescents. The questions were understood by the participants. It was possible to make a final assessment of NSSID based on the information gathered in the CANDI interview. Several of the self-injury methods listed in the CANDI, however, such as using acid, bleach or breaking bones, were not acknowledged by any of the adolescent participants in this sample.

\section{Psychopathology, Traumatic Experiences, and Comparisons Between NSSI Groups}

Of the total sample $(n=29), 51.7 \%$ met criteria for attention deficit hyperactivity disorder, hyperactive/impulsive or inattentive (ADHD/ADD) subtype, and 48.3\% met criteria for depression. Comorbid anxiety disorders (44.8\%) were also common, as were borderline personality disorder (BPD) traits $(41.4 \%)$. The mean total number of comorbid psychiatric diagnoses was $2.31(S D=1.00)$. NSSID was comorbid with several diagnoses, such as depression $(n=7,38.9 \%)$, anxiety disorder $(n=7,38.9 \%)$, borderline traits $(n=8,44.4 \%)$, and ADHD/ADD $(n=10,55.6 \%)$. NSSID also occurred independently of BPD in 10 cases (55.6\%). Adolescents in the NSSI group who did not meet full NSSID criteria also had several comorbidities: depression $(n=7,63.6 \%)$, anxiety disorder $(n=6$, $54.5 \%)$, borderline traits $(n=4,36.4 \%)$, and ADHD/ADD $(n=5$, $45.5 \%)$. There were no significant differences between comorbid diagnoses in the NSSID and the NSSI group. The psychiatric diagnoses for the total sample and respective group (NSSID and NSSI) are presented in Table 5.

Six of the 18 adolescents (33.3\%) in the NSSID group and none $(0.0 \%)$ in the NSSI group had been in inpatient care. This was not a significant difference, but a trend $(p=0.06)$. See Table 5. There were no significant differences between groups regarding psychopathology, such as borderline symptom scores, selfreport or interview assessment, emotion regulation difficulties, alexithymia, or clinician-rated depressive symptoms.

Concerning traumatic experiences, the total number of selfreported negative life events or traumatic symptoms did not differ significantly between the groups (Table 5).

TABLE 5 | Psychopathology, traumatic experiences, and comparisons between NSSI groups.

\begin{tabular}{|c|c|c|c|c|}
\hline Psychiatric diagnoses* & $\begin{array}{c}\text { Total NSSI } \\
\text { sample } \\
(n=29) \\
n(\%)\end{array}$ & $\begin{array}{c}\text { NSSID } \\
(n=18) \\
n(\%)\end{array}$ & $\begin{array}{c}\text { NSSI } \\
(n=11) \\
n(\%)\end{array}$ & $p$ \\
\hline Depression & $14(48.3)$ & $7(38.9)$ & $7(63.6)$ & $0.36+$ \\
\hline Anxiety disorder & $13(44.8)$ & $7(38.9)$ & $6(54.5)$ & $0.47 \dagger$ \\
\hline $\begin{array}{l}\text { Posttraumatic stress } \\
\text { disorder }\end{array}$ & $1(3.4)$ & $1(5.6)$ & $0(0.0)$ & $1.00 \dagger$ \\
\hline Borderline traits & $12(41.4)$ & $8(44.4)$ & $4(36.4)$ & $0.72 \dagger$ \\
\hline Eating disorder & $6(20.7)$ & $3(16.7)$ & $3(27.3)$ & $0.65+$ \\
\hline ADHD/ADD & $15(51.7)$ & $10(55.6)$ & $5(45.5)$ & $0.89+$ \\
\hline High-functioning autism & $3(10.3)$ & $3(16.7)$ & $0(0.0)$ & $0.27 \dagger$ \\
\hline $\mathrm{ODD} / \mathrm{CD}$ & $3(10.3)$ & $3(16.7)$ & $0(0.0)$ & $0.27 \dagger$ \\
\hline $\begin{array}{l}\text { Ever had inpatient } \\
\text { psychiatric care }\end{array}$ & $6(20.7)$ & $6(33.3)$ & $0(0.0)$ & $0.06+$ \\
\hline Mean total diagnoses $m(S D)$ & $2.31(1.00)$ & $2.33(1.08)$ & $2.27(0.90)$ & $0.98 \neq$ \\
\hline SCID-II self-report $m(S D)$ & $5.93(2.83)$ & $5.44(2.98)$ & $6.73(2.49)$ & $0.20 \neq$ \\
\hline SCID-II interview m (SD) & $3.14(2.56)$ & $2.78(2.71)$ & $3.73(2.28)$ & $0.29 \neq$ \\
\hline DERS $m(S D)$ & $123.96(23.25)$ & $123.0(24.76)$ & $125.89(21.15)$ & $0.88 \neq$ \\
\hline TAS-20 m (SD) & $63.22(11.35)$ & $63.56(11.62)$ & $62.56(11.46)$ & $0.96 \neq$ \\
\hline TSCC $m(S D)$ & $62.63(20.70)$ & $59.56(19.46)$ & $68.78(22.88)$ & $0.35 \neq$ \\
\hline LYLES $m(S D)$ & 8.33 (3.63) & $7.78(3.80)$ & $9.44(3.17)$ & $0.44 \neq$ \\
\hline
\end{tabular}

NSSID nonsuicidal self-injury disorder; NSSI, nonsuicidal self-injury; CDRS-R, Children's Depressive Rating Scale-Revised; SCID-II, Structural Clinical Interview for DSM-IV Personality Disorders; LYLES, Linköping Youth Life Event Scale; TSCC, Trauma Symptom Checklist for Children; TAS-20, Toronto Alexithymia Scale; DERS, Difficulties in Emotion Regulation Scale; $A D H D / A D D$, attention deficit hyperactivity disorder/attention deficit disorder; $O D D$, oppositional defiant disorder; $C D$, conduct disorder. * Every patient could have more than one diagnosis. IChi-square with Yates correction. † Fischer's exact test. \# Independent samples t-test (due to small samples analyses were also run with Mann-Whitney U-test with similar results). 


\section{DISCUSSION}

The clinical utility of the NSSID diagnosis was examined, and the feasibility of using semi-structured CANDI to assess diagnosis criteria in a clinical sample of adolescents with NSSI. Although originally developed for adults, CANDI was a helpful measure in assessing NSSID and NSSI characteristics in this clinical adolescent sample, and thus well suited for this purpose. Of the clinical adolescents with NSSI in this sample, $62.1 \%$ met full criteria for NSSID, with criterion $\mathrm{E}$ being least commonly met. Cutting frequency significantly discriminated those with NSSID from those with NSSI not meeting full criteria. Those with NSSID did not differ from those with NSSI not meeting full criteria on other self-injury characteristics or psychopathology. NSSID occurred both independently and together with other psychiatric disorders and suicidality, as did NSSI.

\section{Self-Injury Characteristics}

The NSSID prevalence rate of $62.1 \%$ in this clinical sample of self-injuring adolescents is slightly lower than earlier studies of NSSID in clinical self-injuring adolescents, which have found NSSID rates of $74-78 \%(8,14)$. The present sample was predominately an outpatient sample, while the studies from both Glenn and Klonsky and Washburn and colleagues had more severe inpatient participants who were in treatment specifically for NSSI, which is a possible explanation for the higher prevalence. Other prevalence studies of NSSID in adults have shown result rates ranging between 37 and $46.2 \%$ in selfinjuring clinical college samples and psychiatric outpatient samples, respectively $(22,23)$. The prevalence rates in the current study thus fall somewhere in between, which is tentatively plausible and contributes further empirical adolescent data toward the NSSID diagnosis.

Those not meeting criterion A (13.8\%), despite the presence of five or more NSSI episodes on different days during the past year, were assessed as having too minor a form of self-injury that did not result in bleeding or bruising. CANDI thus identified individuals who did not meet criterion A but had engaged in NSSI during the past 6 months.

Criteria B and $C$ were met by nearly every subject $(96.6 \%$ and $100 \%$ ), which, together with similar earlier findings (20), questions the clinical utility of these criteria. The present study also confirmed previous studies $(22,25)$ concerning criterion E, which had the lowest endorsement (72.4\%). This has also shown to be the case in community samples of adolescents $(11,12)$. Criterion $\mathrm{E}$ is a necessary prerequisite for discriminating those meeting NSSID criteria from those who do not $(21,23)$, since the diagnosis needs to identify those who are impaired by their symptoms. The impairment/distress criterion is thus central to any diagnosis. The clinical utility of this criterion needs to be examined further, however, as many adolescents tend to see their NSSI as helpful rather than distressing (21). In this study the participants tended to attribute their impairment or distress to their comorbid conditions rather than to NSSI per se.

The only significant difference between those with NSSID and those with NSSI was for cutting frequency. Some of the few earlier studies that have investigated the NSSID diagnosis have identified a more serious group concerning NSSI characteristics and psychopathology (23) using the NSSID criteria, but there have also been some inconsistencies concerning the validity and limited clinical utility in clinical samples as the diagnosis is currently formulated $(8,18)$. When data in the current study were examined there was a higher proportion of adolescents with suicidal plans in the NSSID group compared to those without an NSSID diagnosis, which tentatively supports the presence of a somewhat more severe group among those who met all NSSID criteria. Lack of statistical significance is probably due to the small sample size, so interpretations need to be made with caution.

\section{Psychopathology and Traumatic Experiences}

Results from this study confirm earlier research, where NSSID in clinical samples has been shown to exist both together with and independently of different psychiatric conditions and suicidality $(8,15,24)$. This is an important delineation when conceptualizing NSSID, and strengthens the validity of the diagnosis. The most common comorbid conditions in the total sample were ADHD/ADD and depression. Psychopathology did not delimit those with NSSID from those with NSSI not meeting full criteria: depression ( $38.9 \%$ vs $63.6 \%$ ), ADHD (55.6\% vs $45.5 \%)$, anxiety disorders (38.9\% vs $54.5 \%)$, and borderline traits $(44.4 \%$ vs $36.4 \%)$, respectively. Similarly, In-Albon and colleagues did not find diagnostic differences between NSSID and a group with NSSID not meeting full criteria (25). An earlier study that used CANDI in a community sample of young adults with recurrent NSSI (23) did however find some diagnostic differences between the two groups. Those with NSSID had more BPD, bipolar disorder, PTSD, social anxiety disorder, and alcohol dependence compared to those not meeting full criteria. Some diagnostic differences were also found by Kiekens et al. (14), who compared NSSID with a sub-threshold NSSID group. These differences between studies might be due to different samples (adolescents compared to young adults; psychiatric compared to community sample with recurrent NSSI).

In the present sample there were no differences between the NSSID group and the NSSI group on measures of alexithymia or difficulties with emotion regulation. Both groups reported high scores on these measures. In an earlier study by Lüdtke et al. (27) on an inpatient adolescent sample, alexithymia was a significant predictor of NSSID. Difficulties with identifying and describing feelings could potentially increase endorsement of criteria $\mathrm{B}$ and $\mathrm{C}$ in the NSSID diagnosis.

There was also a trend for those with NSSID (33.3\%) in this study to have more experience of inpatient care than those with NSSI (0.0\%), which is an indicator of severity.

Traumatic symptoms, as assessed with TSCC, were high in the present total sample $(M=62.63, S D=20.70)$ compared to a previous study of a clinical $(M=52.6, S D=23.9)$ and normative group $(M=30.4, S D=18.7)$ of Swedish adolescents (40). Trauma experience did not separate those with NSSID from those with 
NSSI, nor did negative life events. This is in line with the earlier study by Lüdtke and colleagues (27), who found that childhood maltreatment and dissociation did not predict a diagnosis of NSSID.

A potential interpretation of the results in the present study could be that the NSSID diagnosis is less useful in discriminating between groups in self-injuring clinical samples with several comorbidities and a high level of psychopathology, compared to community/college samples or clinical samples which includes participants who do not self-injure. This interpretation is similar to the findings of Washburn and colleagues (8). Kiekens and colleagues (14) recently examined NSSID in a large college sample and found a dose-response relationship between NSSI recency and severity and mental disorders and suicidality, where the strength of the association was smaller in the sub-threshold NSSID group compared to those meeting full NSSID criteria.

One assumption could be that since all the individuals in clinical samples are in a severe condition, often with trauma experiences, comorbidity, suicidality, and functional impairment, NSSID as currently defined will not discriminate as clearly within clinical relative to community samples. Thus the clinical utility of the NSSID diagnosis is more limited in identifying a group in need of intervention in this context. Notwithstanding, diagnostic criteria and cut-offs need to be the same regardless of type of sample. If cut-offs were based on empirically derived thresholds in clinical samples, the cutoffs would be higher and would then identify a much more severe group. In that case, there would potentially be a risk of missing NSSID sub-threshold individuals who are also struggling with NSSI and require treatment to avoid an escalating negative trajectory. The NSSID diagnosis would, however, perhaps benefit from different cut-offs based on severity ratings, similar to depression, for example, with minor, moderate and severe conditions. This could potentially increase utility in clinical samples. One such attempt was made by Muehlenkamp et al. (18), who investigated differences between groups with low (1-4), moderate (5-24) and high ( $\geq 25)$ NSSI frequency.

This study contributes important information on the use of a structured clinical measure to assess prevalence rates of NSSID in clinical adolescents, in addition to self-report measures of the criteria in community samples. Although this study focused on a previously somewhat neglected sample in the research area of NSSID, the study has some limitations that need to be addressed. First, the sample size was small with potential type II errors in the statistical subgroup analyses. Also, the multiple comparisons could lead to type I errors. Secondly, the cross-sectional design prevents longitudinal or causal conclusions, such as predicting risk on the basis of the NSSID diagnosis. Thirdly, no validated cut-off values for CANDI were reported, hence the interpretation of the clinical utility of the CANDI is limited. Fourthly, since only girls were included in the study, the findings are restricted in generalizability to other samples. Fifthly, the age group was homogeneous and therefore the feasibility and clinical utility of CANDI for a younger age group cannot be assessed. Sixthly, no other measure of NSSI was used and thus concurrent validity could not be examined. The diagnosis of NSSID was made by one person and inter-rater reliability is therefore lacking.

The NSSID diagnosis is a potential facilitator in identifying risk and planning treatment. More studies investigating the validity of the criteria in larger clinical samples of adolescents are needed. Furthermore, the clinical utility of the diagnosis, and the potential to discriminate between those meeting full criteria NSSID disorder and those who do not, need to be examined further in order not to over-pathologize the behavior. A prospective area for research could be predictively examining whether those who are identified with NSSID are at higher risk of future self-injury, general psychopathology or a high consumption of health care. Results in this field would contribute important data toward establishing clinical utility.

To conclude, CANDI was a feasible tool to evaluate NSSI as an independent disorder in this psychiatric outpatient sample of adolescents. The NSSID diagnosis discriminated between those with and without NSSID in this NSSI sample by cutting frequency, and possibly by inpatient care and suicidal plans, where those with NSSID were a more severe group. However, patients with NSSI (sub-threshold) not meeting full NSSID criteria showed similar levels of psychopathology and NSSI characteristics as patients with NSSID in this clinical sample.

\section{DATA AVAILABILITY STATEMENT}

The datasets generated for this study will not be made publicly available. We do not have participants' consent to share raw data publicly. The data that has been used is confidential and individual participants' could potentially be identified if the dataset is shared.

\section{ETHICS STATEMENT}

The study involved human participants and was reviewed and approved by the Regional Ethical Board of Linköping (Dnr 2015/ 273-31; 2016/224-32). Written informed consent to participate in this study was provided by the participants and the participants' legal guardian/next of kin.

\section{AUTHOR CONTRIBUTIONS}

MZ, IP, LM, and PG designed research. MZ performed research. MZ analyzed data and made tables. MZ, IP, LM, and PG wrote the paper.

\section{FUNDING}

This research was supported by The Swedish Research Council (538-2013-7434) and the ALF Grants, Östergötland County (LIO-535931; LIO-520131). 


\section{REFERENCES}

1. Nock MK. Self-injury. Annu Rev Clin Psychol (2010) 6:339-63. doi: 10.1146/ annurev.clinpsy.121208.131258

2. Klonsky ED, Muehlenkamp JJ. Self-injury: a research review for the practitioner. J Clin Psychol (2007) 63(11):1045-56. doi: 10.1002/jclp.20412

3. Muehlenkamp JJ, Claes L, Havertape L, Plener PL. International prevalence of non-suicidal self-injury and deliberate self-harm. Child Adolesc Psychiatry Ment Health (2012) 6, 1-9. doi: 10.1186/1753-2000-6-10

4. Muehlenkamp JJ. Self-injurious behavior as a separate clinical syndrome. Am J Orthopsychiatry (2005) 75(2):324-33. doi: 10.1037/0002-9432.75.2.324

5. Shaffer D, Jacobson C. Proposal to the DSMV childhood disorder and mood disorder work groups to include non-suicidal self-injury (NSSI) as a DSM-V disorder. Am Psychiatr Assoc (2009).

6. Zetterqvist M. The DSM-5 diagnosis of nonsuicidal self-injury disorder: a review of the empirical literature. Child Adolesc Psychiatry Ment Health (2015) 9:31. doi: 10.1186/s13034-015-0062-7

7. American Psychiatric Association. Diagnostic and statistical manual of mental disorders (5th ed.). Washington DC: American Psychiatric Publishing (2013). doi: 10.1176/appi.books.9780890425596

8. Washburn JJ, Potthoff LM, Juzwin KR, Styer DM. Assessing DSM-5 nonsuicidal self-injury disorder in a clinical sample. psychol Assess (2015) 27(1):31-41. doi: 10.1037/pas0000021

9. Selby EA, Kranzler A, Fehling KB, Panza E. Nonsuicidal self-injury disorder: the path to diagnostic validity and final obstacles. Clin psychol Rev (2015) 38:79-91. doi: 10.1016/j.cpr.2015.03.003

10. Albores-Gallo L, Mendez-Santos JL, Xochitl-Garcia Luna A, DelgadilloGonzalez Y, Chavez-Flores CI, Martinez OL. Nonsuicidal self-injury in a community sample of older children and adolescents of Mexico City. Actas Españolas Psiquiatría (2014) 42(4):159-68.

11. Buelens T, Luyckx K, Kiekens G, Gandhi A, Muehlenkamp JJ, Claes L. Investigating the DSM-5 criteria for non-suicidal self-injury disorder in a community sample of adolescents. J Affect Disord (2020) 260:314-22. doi: 10.1016/j.jad.2019.09.009

12. Zetterqvist M, Lundh LG, Dahlstrom O, Svedin CG. Prevalence and function of non-suicidal self-injury (NSSI) in a community sample of adolescents, using suggested DSM-5 criteria for a potential NSSI disorder. J Abnormal Child Psychol (2013) 41(5):759-73. doi: 10.1007/s10802-013-9712-5

13. Benjet C, Gonzalez-Herrera I, Castro-Silva E, Mendez E, Borges G, Casanova L, et al. Non-suicidal self-injury in Mexican young adults: prevalence, associations with suicidal behavior and psychiatric disorders, and DSM-5 proposed diagnostic criteria. J Affect Disord (2017) 215:1-8. doi: 10.1016/ j.jad.2017.03.025

14. Kiekens G, Hasking P, Claes L, Mortier P, Auerbach RP, Boyes M, et al. The DSM5 nonsuicidal self-injury disorder among incoming college students: prevalence and associations with 12-month mental disorders and suicidal thoughts and behaviors. Depression Anxiety (2018) 35(7):629-37. doi: 10.1002/da.22754

15. Glenn CR, Klonsky ED. Nonsuicidal self-injury disorder: an empirical investigation in adolescent psychiatric patients. J Clin Child Adolesc Psychol (2013) 42(4):496-507. doi: 10.1080/15374416.2013.794699

16. Ammerman BA, Jacobucci R, McCloskey MS. Reconsidering important outcomes of the nonsuicidal self-injury disorder diagnostic criterion A. J Clin Psychol (2019) 75(6):1084-97. doi: 10.1002/jclp.22754

17. Muehlenkamp JJ, Brausch AM. Reconsidering criterion A for the diagnosis of non-suicidal self-injury disorder. J Psychopathol Behav Assess (2016) 38 (4):547-58. doi: 10.1007/s10862-016-9543-0

18. Muehlenkamp JJ, Brausch AM, Washburn JJ. How much is enough? Examining frequency criteria for NSSI disorder in adolescent inpatients. $J$ Consult Clin Psychol (2017) 85(6):611-9. doi: 10.1037/ccp0000209

19. Plener PL, Kapusta ND, Kölch MG, Kaess M, Brunner R. Non-suicidal selfinjury as autonomous diagnosis-implications for research and clinic of the DSM-5 proposal to establish the diagnosis of non-suicidal self-injury in adolescents. Z Kinder Jugendpsychiatry Psychother (2012) 40:113-120. doi: 10.1024/1422-4917/a000158

20. Brausch AM, Muehlenkamp JJ, Washburn JJ. Nonsuicidal self-injury disorder: does criterion B add diagnostic utility? Psychiatry Res (2016) 244:179-84. doi: 10.1016/j.psychres.2016.07.025
21. Zetterqvist M. Nonsuicidal self-injury in adolescents: characterization of the disorder and the issue of distress and impairment. Suicide Life-Threat Behav (2017) 47(3):321-35. doi: 10.1111/sltb.12283

22. Odelius CB, Ramklint M. Clinical utility of proposed non-suicidal self-injury diagnosis-a pilot study. Nordic J Psychiatry (2014) 68(1):66-71. doi: 10.3109/ 08039488.2013.775340

23. Gratz KL, Dixon-Gordon KL, Chapman AL, Tull MT. Diagnosis and characterization of DSM-5 nonsuicidal self-injury disorder using the clinician-administered nonsuicidal self-injury disorder index. Assessment (2015) 22(5):527-39. doi: 10.1177/1073191114565878

24. Selby EA, Bender TW, Gordon KH, Nock MK, Joiner TEJr.. Non-suicidal selfinjury (NSSI) disorder: a preliminary study. Pers Disord: Theory Res Treat (2012) 3(2):167-75. doi: 10.1037/a0024405

25. In-Albon T, Ruf C, Schmid M. Proposed diagnostic criteria for the DSM-5 of nonsuicidal self-injury in female adolescents: diagnostic and clinical correlates. Psychiatry J (2013). 2013(159208):1-12. doi: 10.1155/2013/159208

26. Perez J, Venta A, Garnaat S, Sharp C. The difficulties in emotion regulation scale: factor structure and association with nonsuicidal self-injury in adolescent inpatients. J Psychopathol Behav Assess (2012) 34(3):393-404. doi: 10.1007/s10862-012-9292-7

27. Lüdtke J, In-Albon T, Michel C, Schmid M. Predictors for DSM-5 nonsuicidal self-injury in female adolescent inpatients: the role of childhood maltreatment, alexithymia, and dissociation. Psychiatry Res (2016) 239:34652. doi: 10.1016/j.psychres.2016.02.026

28. Regier DA, Narrow WE, Clarke DE, Kraemer HC, Kuramoto SJ, Kuhl EA, et al. DSM-5 field trials in the United States and Canada, Part II: test-retest reliability of selected categorical diagnoses. Am J Psychiatry (2013) 170(1):5970. doi: 10.1176/appi.ajp.2012.12070999

29. Bjureberg J, Sahlin H, Hedman-Lagerlof E, Gratz KL, Tull MT, Jokinen J, et al. Extending research on Emotion Regulation Individual Therapy for Adolescents (ERITA) with nonsuicidal self-injury disorder: open pilot trial and mediation analysis of a novel online version. BMC Psychiatry (2018) 18 (1):326. doi: 10.1186/s12888-018-1885-6

30. Bjureberg J, Sahlin H, Hellner C, Hedman-Lagerlof E, Gratz KL, Bjarehed J, et al. Emotion regulation individual therapy for adolescents with nonsuicidal self-injury disorder: a feasibility study. BMC Psychiatry (2017) 17(1):411. doi: 10.1186/s12888-017-1527-4

31. Perini I, Gustafsson PA, Hamilton JP, Kämpe R, Mayo L, Heilig M, et al. Brain-based classification of negative social bias in adolescents with nonsuicidal self-injury: findings from simulated online social interaction. EClin Med (2019) 13:81-90 doi: 10.1016/j.eclinm.2019.06.016

32. Nisbett RE, Wilson TD. Telling more than we can know: verbal reports on mental processes. psychol Rev (1977) 84:231-59. doi: 10.1037/0033295X.84.3.231

33. Bresin K, Schoenleber M. Gender differences in the prevalence of nonsuicidal self-injury: a meta-analysis. Clin psychol Rev (2015) 38:55-64. doi: 10.1016/ j.cpr.2015.02.009

34. Nock MK, Holmberg Eb Fau - Photos VI, Photos Vi Fau - Michel BD, Michel BD. Self-Injurious thoughts and behaviors interview: development, reliability, and validity in an adolescent sample. psychol Assess (2007) 19:309-17. doi: $10.1037 / 1040-3590.19 .3 .309$

35. Kaufman J, Birmaher B, Brent D, Rao U, Flynn C, Moreci P, et al. Schedule for affective disorders and schizophrenia for school-age children-present and lifetime version (K-SADS-PL): initial reliability and validity data. J Am Acad Child Adolesc Psychiatry (1997) 36:980-8. doi: 10.1097/00004583-199707000-00021

36. Gratz KL, Gunderson JG. Preliminary data on an acceptance-based emotion regulation group intervention for deliberate self-harm among women with borderline personality disorder. Behav Ther (2006) 37(1):25-35. doi: 10.1016/ j.beth.2005.03.002

37. Taylor GJ, Doody K. Verbal measures of alexithymia: what do they measure. Psychother Psychosom (1985) 43(1):32-7. doi: 10.1159/000287855

38. First MB, Gibbon M, Spitzer RL, Williams JBW, Benjamin LS. Structured Clinical Interview for DSM-IV Axis II Personality Disorders, (SCID-II). Washington, D.C: American Psychiatric Press, Inc. (1997).

39. Briere J. Trauma symptom checklist for children (TSCC) professional manual. Odessa, FL: Psychological Assessment Resources (1996). doi: $10.1037 / \mathrm{t} 06631-000$ 
40. Nilsson D, Wadsby M, Svedin CG. The psychometric properties of the Trauma Symptom Checklist for Children (TSCC) in a sample of Swedish children. Child Abuse Negl (2008) 32:627-36. doi: 10.1016/j.chiabu.2007.09.009

41. Nilsson D, Gustafsson PE, Larsson JL, Svedin C-G. Evaluation of the linköping youth life experience scale. J Nervous Ment Dis (2010) 198:768-74. doi: 10.1097/NMD.0b013e3181f4acb6

42. Wechsler D. The Wechsler intelligence scale for children-fourth edition. London: Pearson (2003). doi: 10.1037/t15174-000

43. Wechsler $\mathrm{D}$. The Wechsler adult intelligence scale-fourth edition. London: Pearson (2008). doi: 10.1037/t15169-000
Conflict of Interest: The authors declare that the research was conducted in the absence of any commercial or financial relationships that could be construed as a potential conflict of interest.

Copyright $\odot 2020$ Zetterqvist, Perini, Mayo and Gustafsson. This is an open-access article distributed under the terms of the Creative Commons Attribution License (CC $B Y$ ). The use, distribution or reproduction in other forums is permitted, provided the original author(s) and the copyright owner(s) are credited and that the original publication in this journal is cited, in accordance with accepted academic practice. No use, distribution or reproduction is permitted which does not comply with these terms. 